\title{
The genome sequence of the German wasp, Vespula germanica
}

\section{(Fabricius, 1793) [version 1; peer review: 2 approved]}

\author{
Liam M. Crowley (iD), \\ University of Oxford and Wytham Woods Genome Acquisition Lab, \\ Darwin Tree of Life Barcoding collective, \\ Wellcome Sanger Institute Tree of Life programme, \\ Wellcome Sanger Institute Scientific Operations: DNA Pipelines collective, \\ Tree of Life Core Informatics collective, Darwin Tree of Life Consortium \\ ${ }^{1}$ Department of Zoology, University of Oxford, Oxford, UK
}

V1 First published: 15 Feb 2022, 7:60

https://doi.org/10.12688/wellcomeopenres.17703.1

Latest published: 15 Feb 2022, 7:60

https://doi.org/10.12688/wellcomeopenres.17703.1

\section{Abstract}

We present a genome assembly from an individual female Vespula germanica (the German wasp; Arthropoda; Insecta; Hymenoptera; Vespidae). The genome sequence is 206 megabases in span. The majority of the assembly (98.55\%) is scaffolded into 25 chromosomal pseudomolecules. The mitochondrial genome was also assembled and is 18.0 kilobases in length. Annotation of the genome assembly on Ensembl has identified 12,361 protein-coding genes.

\section{Keywords}

Vespula germanica, German wasp, genome sequence, chromosomal, Hymenoptera

This article is included in the Tree of Life gateway.

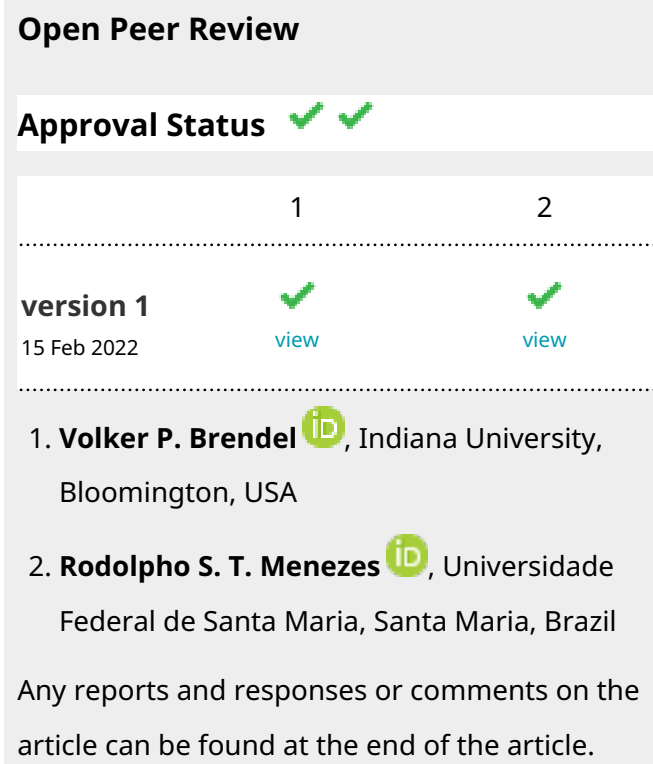

1

2

version 1

15 Feb 2022

view

view

1. Volker P. Brendel ID, Indiana University, Bloomington, USA

2. Rodolpho S. T. Menezes ID, Universidade Federal de Santa Maria, Santa Maria, Brazil Any reports and responses or comments on the article can be found at the end of the article. 
Corresponding author: Darwin Tree of Life Consortium (mark.blaxter@sanger.ac.uk)

Author roles: Crowley LM: Investigation, Resources, Writing - Original Draft Preparation;

Competing interests: No competing interests were disclosed.

Grant information: This work was supported by Wellcome through core funding to the Wellcome Sanger Institute (206194) and the Darwin Tree of Life Discretionary Award (218328).

The funders had no role in study design, data collection and analysis, decision to publish, or preparation of the manuscript.

Copyright: (c) 2022 Crowley LM et al. This is an open access article distributed under the terms of the Creative Commons Attribution License, which permits unrestricted use, distribution, and reproduction in any medium, provided the original work is properly cited.

How to cite this article: Crowley LM, University of Oxford and Wytham Woods Genome Acquisition Lab, Darwin Tree of Life Barcoding collective et al. The genome sequence of the German wasp, Vespula germanica (Fabricius, 1793) [version 1; peer review: 2 approved] Wellcome Open Research 2022, 7:60 https://doi.org/10.12688/wellcomeopenres.17703.1

First published: 15 Feb 2022, 7:60 https://doi.org/10.12688/wellcomeopenres.17703.1 


\section{Species taxonomy}

Eukaryota; Metazoa; Ecdysozoa; Arthropoda; Hexapoda; Insecta; Pterygota; Neoptera; Endopterygota; Hymenoptera; Apocrita; Aculeata; Vespoidea; Vespidae; Vespinae; Vespula; Vespula germanica (Fabricius, 1793) (NCBI:txid30212).

\section{Background}

The German wasp, Vespula germanica, is a common and widespread species of social wasp throughout the palearctic. It has been introduced and become established in North America, South America, South Africa, Australia and New Zealand (Archer, 1998), where in some instances it has become a significant invasive species (Beggs et al., 2011; Eloff et al., 2020; Masciocchi \& Corley, 2013). It is one of the most common and widespread social wasps in the UK, although populations have declined markedly since the 1970s (Archer, 2001). It is found throughout a wide variety of habitats, including urban areas. This species is eusocial, living in colonies with a reproductive queen $(16-19 \mathrm{~mm})$, sterile workers $(12-14 \mathrm{~mm})$ and reproductive males (14-17 mm) (Archer \& Turner, 2014). Adults are strikingly marked yellow and black, with clear yellow genae that distinguish it from the very similar Vespula vulgaris. The clypeal markings varyingly consist of an incomplete dorso-ventral stripe plus two spots or three spots, unlike the anchor-like marking that is typical of $V$. vulgaris.

Colonies are annual in the UK, typically producing up to around 8000 cells (Archer \& Turner, 2014). Nests are mostly constructed underground, particularly within old rodent holes, but can also be found in enclosed aerial situations such as roof spaces, sheds and thick hedges (Archer, 1998). Nests are constructed out of a paper-like substance produced from macerated wood fibres mixed with saliva. The nest consists of hexagonal cells arranged into eight to nine combs, covered by a nest envelope. The nest envelope of this species is grey in colour due to the well-weathered wood used to make the pulp.

Overwintered queens typically emerge from overwintering diapause around mid-March, nest founding occurs in May, and the first workers appear from early June. Males and new queens are produced from August, and mating occurs in September. Queens are typically polyandrous (Goodisman et al., 2002). Nests usually persist until around November, although some may last through the winter until early spring. There is large variation in population sizes between years, with cyclical abundance and scarcity of individuals (Archer, 1985).

This species is generalist opportunistic predator and scavenger, with workers preying on a wide range of insect and other arthropod species and scavenging from large protein sources, such as carrion (D'adamo \& Lozada, 2007). Food is malaxated before being carried back to the nest to be fed to the developing brood. Adults feed on carbohydrate rich substances including nectar, sap, honeydew and secretions from the larvae. The propensity of adults to visit flowers, particularly shallow blooms such as ivy (Hedera helix) and umbellifers, means this species may act an important pollinator. Workers have been shown to exhibit limited temporal polyethism, with nest work, pulp foraging, carbohydrate foraging, and protein foraging sequentially performed by workers as they age (Hurd et al., 2007).

\section{Genome sequence report}

The genome was sequenced from a single female $V$. germanica collected from Wytham Woods, Oxfordshire (biological vicecounty: Berkshire), UK (latitude 51.770, longitude -1.339). A total of 72-fold coverage in Pacific Biosciences singlemolecule long reads and 181-fold coverage in 10X Genomics read clouds were generated. Primary assembly contigs were scaffolded with chromosome conformation Hi-C data. Manual assembly curation corrected $13 \mathrm{missing} / \mathrm{misjoins}$ and removed one haplotypic duplication, reducing the scaffold number by $21.74 \%$, and increasing the scaffold N50 by $9.19 \%$.

The final assembly has a total length of $206 \mathrm{Mb}$ in 36 sequence scaffolds with a scaffold N50 of $9.4 \mathrm{Mb}$ (Table 1). Of the assembly sequence, $98.55 \%$ was assigned to 25 chromosomal-level scaffolds (numbered by sequence length) (Figure 1-Figure 4; Table 2). The assembly has a BUSCO v5.1.2 (Manni et al., 2021) completeness of $96.4 \%$ (single $96.1 \%$, duplicated $0.3 \%$ ) using the hymenoptera_odb10 reference set $(n=5991)$. While not fully phased, the assembly deposited is of one haplotype. Contigs corresponding to the second haplotype have also been deposited.

\section{Genome annotation report}

The iyVesGerm1.1 genome assembly has been annotated using the Ensembl rapid annotation pipeline (Table 1; https://rapid.ensembl. org/Vespula_germanica_GCA_905340365.1/). The resulting annotation includes 34,067 transcribed RNAs from 12,361 protein-coding and 4,967 non-coding genes. There are 2.26 coding transcripts per gene and 6.94 exons per transcript.

\section{Methods}

Sample acquisition and DNA extraction

A single female $V$. germanica (iyVesGerm1) was collected from Wytham Woods, Oxfordshire (biological vice-county: Berkshire), UK (latitude 51.770, longitude -1.339) by Liam Crowley, University of Oxford, using a net. The sample was identified by the same individual and snap-frozen on dry ice. Unfortunately, no image of the sequenced specimen is available.

DNA was extracted from head/thorax tissue of iyVeGerm1 at the Wellcome Sanger Institute (WSI) Scientific Operations core from the whole organism using the Qiagen MagAttract HMW DNA kit, according to the manufacturer's instructions. RNA was extracted from remaining head/thorax tissue of iyVesGerm 1 in the Tree of Life Laboratory at the WSI using TRIzol (Invitrogen), according to the manufacturer's instructions. RNA was then eluted in $50 \mu \mathrm{l}$ RNAse-free water and 
Table 1. Genome data for Vespula germanica, iyVesGerm1.1.

\begin{tabular}{|c|c|}
\hline \multicolumn{2}{|l|}{ Project accession data } \\
\hline Assembly identifier & iyVesGerm1.1 \\
\hline Species & Vespula germanica \\
\hline Specimen & iyVesGerm1 \\
\hline NCBI taxonomy ID & NCBI:txid30212 \\
\hline BioProject & PRJEB43741 \\
\hline BioSample ID & SAMEA7520501 \\
\hline Isolate information & Female, head/thorax \\
\hline \multicolumn{2}{|l|}{ Raw data accessions } \\
\hline PacificBiosciences SEQUEL II & ERR6635596 \\
\hline 10X Genomics Illumina & ERR6054558-ERR6054561 \\
\hline Hi-C Illumina & ERR6054562-ERR6054564 \\
\hline PolyA RNA-Seq Illumina & ERR6286717 \\
\hline \multicolumn{2}{|l|}{ Genome assembly } \\
\hline Assembly accession & GCA_905340365.1 \\
\hline Accession of alternate haplotype & GCA_905340295.1 \\
\hline Span (Mb) & 206 \\
\hline Number of contigs & 56 \\
\hline Contig N50 length (Mb) & 6.6 \\
\hline Number of scaffolds & 36 \\
\hline Scaffold N50 length (Mb) & 9.4 \\
\hline Longest scaffold (Mb) & 20.4 \\
\hline BUSCO* genome score & $\begin{array}{l}\text { C:96.4\%[S:96.1\%,D:0.3\%], } \\
\text { F:0.9\%,M:2.7\%,n:5991 }\end{array}$ \\
\hline \multicolumn{2}{|l|}{ Genome annotation } \\
\hline Number of protein-coding genes & 12,361 \\
\hline Average length of coding sequence (bp) & $1,553.05$ \\
\hline Average number of exons per transcript & 6.94 \\
\hline Average exon size (bp) & 331.04 \\
\hline Average intron size (bp) & $1,355.58$ \\
\hline
\end{tabular}

*BUSCO scores based on the hymenoptera_odb10 BUSCO set using v5.1.2. C= complete [S= single copy, $\mathrm{D}=$ duplicated], $\mathrm{F}=$ fragmented, $\mathrm{M}=$ =missing, $\mathrm{n}=$ number of orthologues in comparison. A full set of BUSCO scores is available at https:// blobtoolkit.genomehubs.org/view/CAJPHY01/dataset/CAJPHY01/busco.

its concentration assessed using a Nanodrop spectrophotometer and Qubit Fluorometer using the Qubit RNA Broad-Range (BR) Assay kit. Analysis of the integrity of the RNA was done using Agilent RNA 6000 Pico Kit and Eukaryotic Total RNA assay.

\section{Sequencing}

Pacific Biosciences $\mathrm{HiFi}$ circular consensus and 10X Genomics Chromium read cloud sequencing libraries were constructed according to the manufacturers' instructions. Poly(A) RNA-Seq libraries were constructed using the NEB Ultra II 


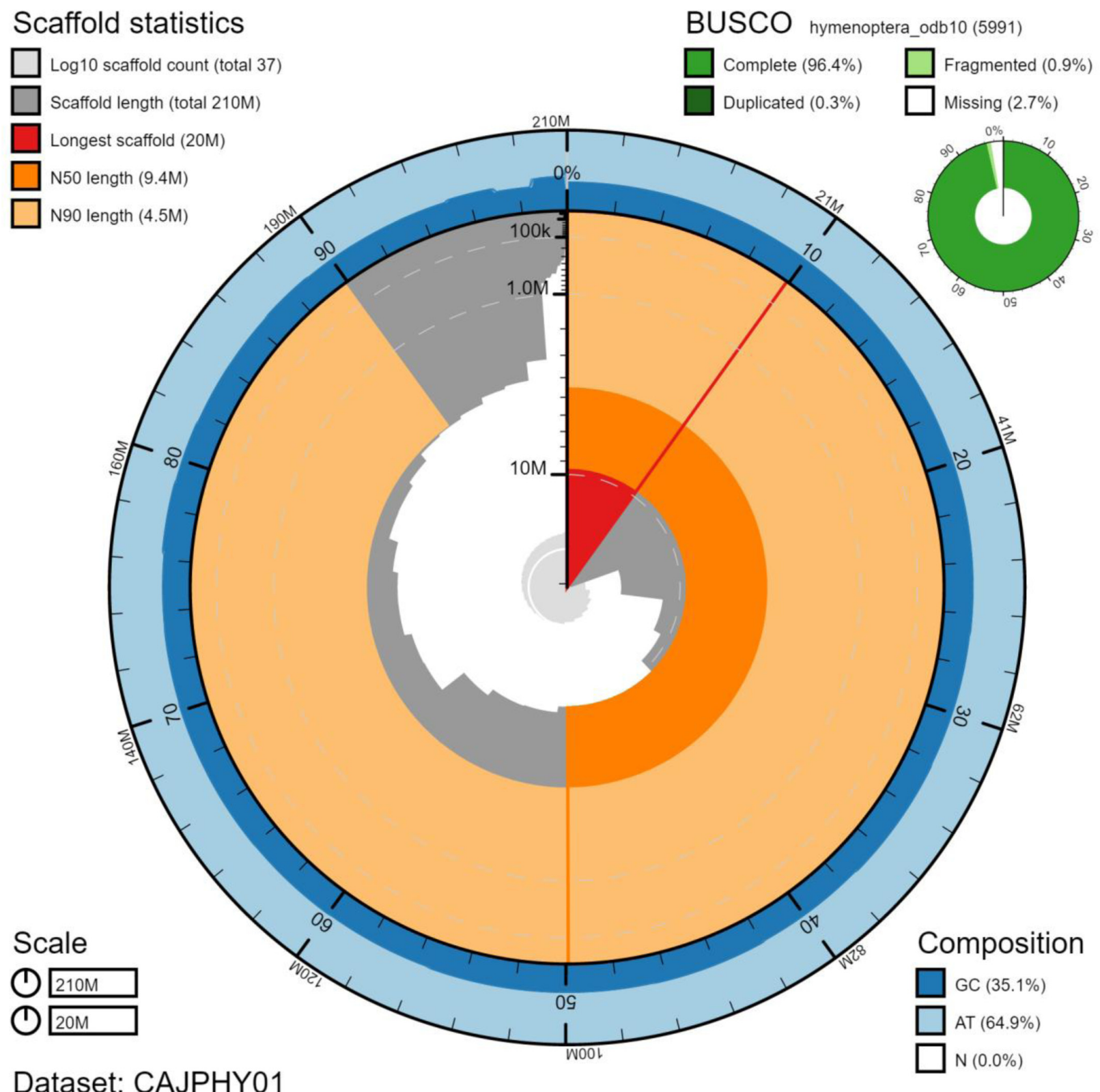

Figure 1. Genome assembly of Vespula germanica, iyVesGerm1.1: metrics. The BlobToolKit Snailplot shows N50 metrics and BUSCO gene completeness. The main plot is divided into 1,000 size-ordered bins around the circumference with each bin representing $0.1 \%$ of the 205,789,424 bp assembly. The distribution of scaffold lengths is shown in dark grey with the plot radius scaled to the longest scaffold present in the assembly (20,389,060 bp, shown in red). Orange and pale-orange arcs show the N50 and N90 scaffold lengths (9,441,317 and $4,478,551$ bp), respectively. The pale grey spiral shows the cumulative scaffold count on a log scale with white scale lines showing successive orders of magnitude. The blue and pale-blue area around the outside of the plot shows the distribution of GC, AT and N percentages in the same bins as the inner plot. A summary of complete, fragmented, duplicated and missing BUSCO genes in the hymenoptera_odb10 set is shown in the top right. An interactive version of this figure is available at https://blobtoolkit.genomehubs.org/view/CAJPHY01/dataset/ CAJPHY01/snail.

RNA Library Prep kit. Sequencing was performed by the Scientific Operations core at the Wellcome Sanger Institute on Pacific Biosciences SEQUEL II (HiFi), Illumina HiSeq X (10X) and Illumina HiSeq 4000 (RNA-Seq) instruments. Hi-C data were generated from head/thorax tissue of iyVesGerm1 using the Arima v2 kit and sequenced on HiSeq X. 


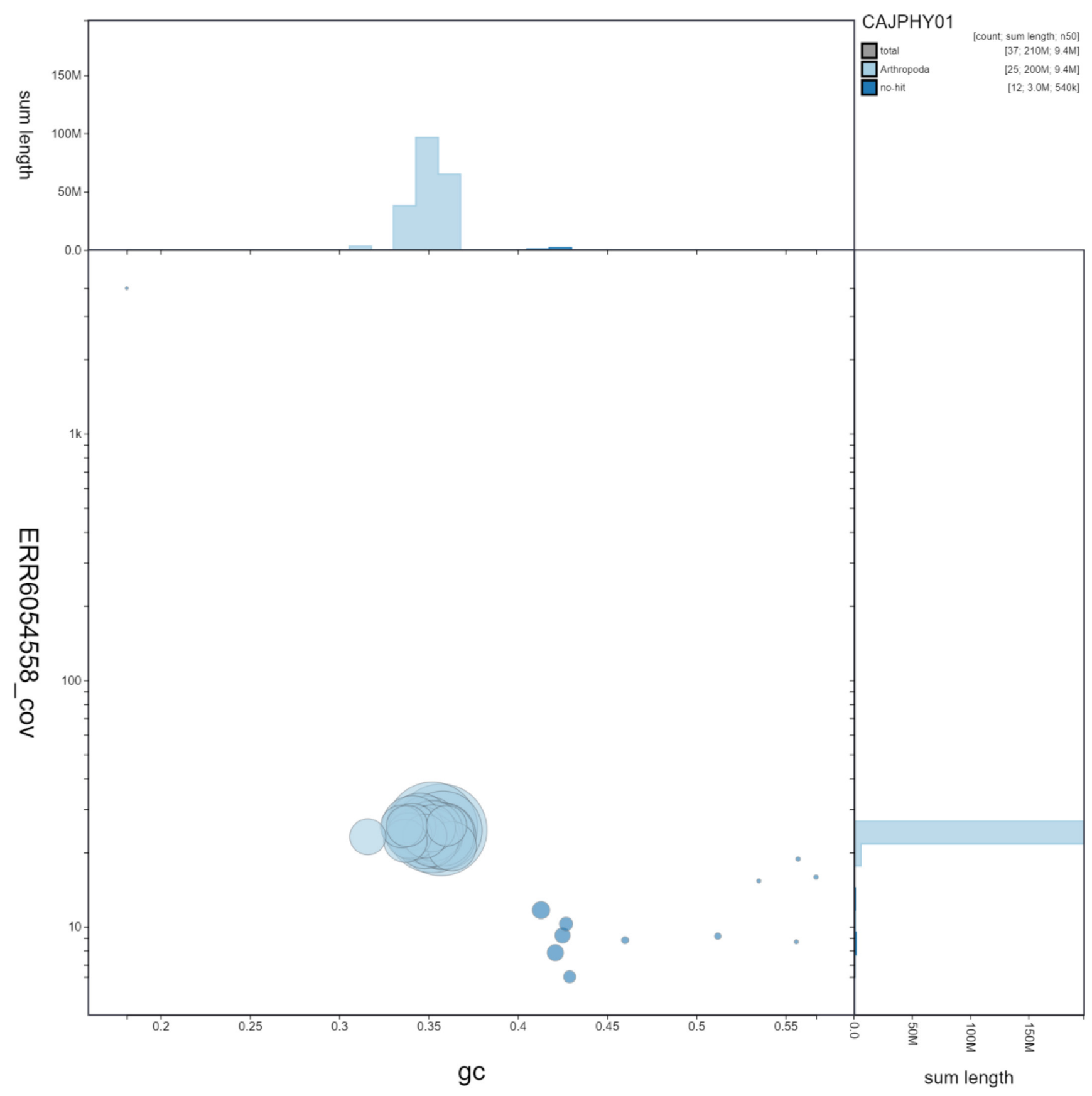

Figure 2. Genome assembly of Vespula germanica, iyVesGerm1.1: GC coverage. BlobToolKit GC-coverage plot. Scaffolds are coloured by phylum. Circles are sized in proportion to scaffold length. Histograms show the distribution of scaffold length sum along each axis. An interactive version of this figure is available at https://blobtoolkit.genomehubs.org/view/CAJPHY01/dataset/CAJPHY01/blob.

\section{Genome assembly}

Assembly was carried out with Hifiasm (Cheng et al., 2021). Haplotypic duplication was identified and removed with purge_dups (Guan et al., 2020). One round of polishing was performed by aligning $10 \mathrm{X}$ Genomics read data to the assembly with longranger align, calling variants with freebayes (Garrison \& Marth, 2012). The assembly was then scaffolded with Hi-C data (Rao et al., 2014) using SALSA2 (Ghurye et al., 2019). The assembly was checked for contamination and corrected using the gEVAL system (Chow et al., 2016) as described 


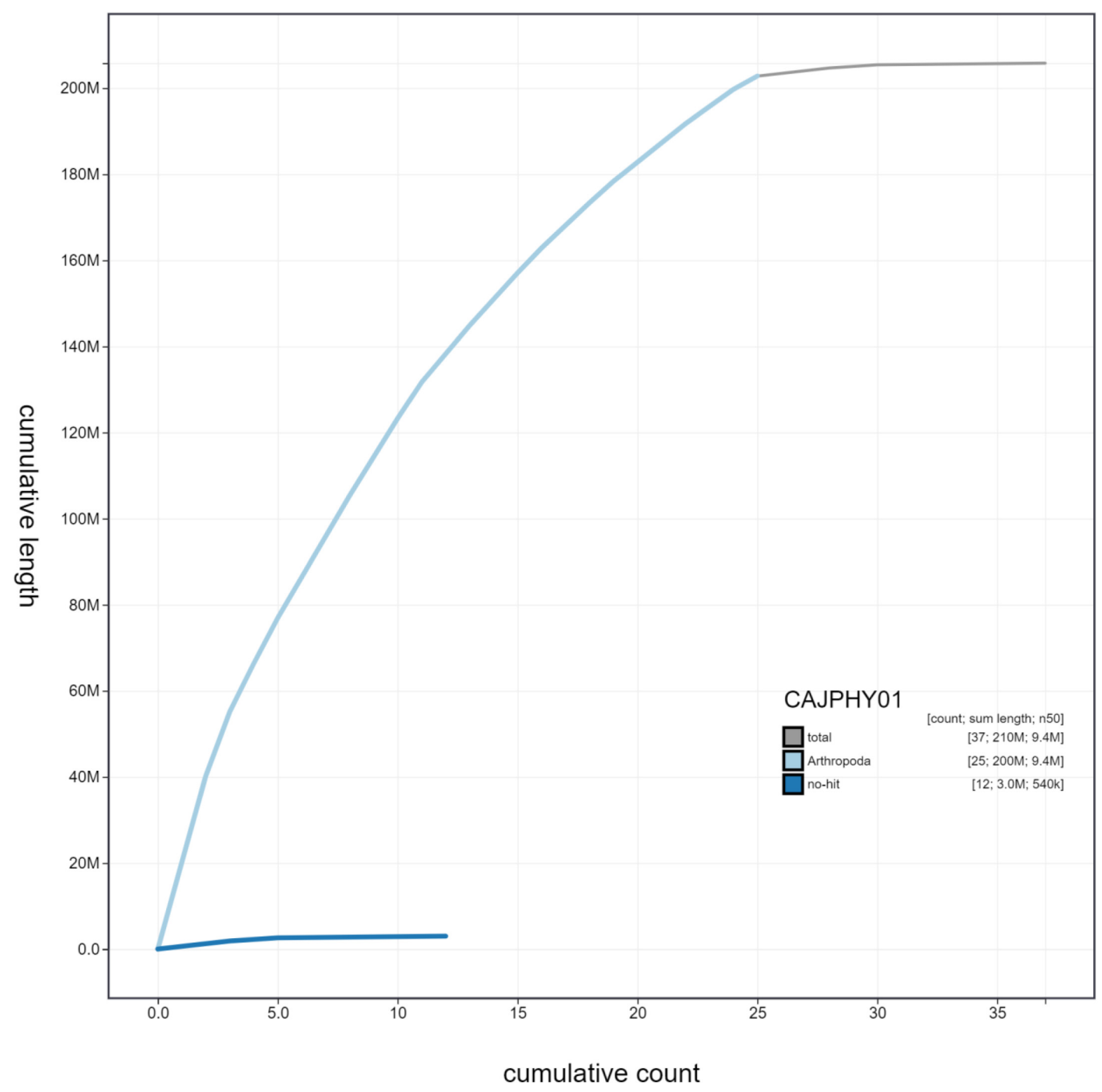

Figure 3. Genome assembly of Vespula germanica, iyVesGerm1.1: cumulative sequence. BlobToolKit cumulative sequence plot. The grey line shows cumulative length for all scaffolds. Coloured lines show cumulative lengths of scaffolds assigned to each phylum using the buscogenes taxrule. An interactive version of this figure is available at https://blobtoolkit.genomehubs.org/view/CAJPHY01/dataset/ CAJPHY01/cumulative.

previously (Howe et al., 2021). Manual curation was performed using gEVAL, HiGlass (Kerpedjiev et al., 2018) and Pretext. The mitochondrial genome was assembled using MitoHiFi (Uliano-Silva et al., 2021), which performed annotation using
MitoFinder (Allio et al., 2020). The genome was analysed and BUSCO scores generated within the BlobToolKit environment (Challis et al., 2020). Table 3 contains a list of all software tool versions used, where appropriate. 


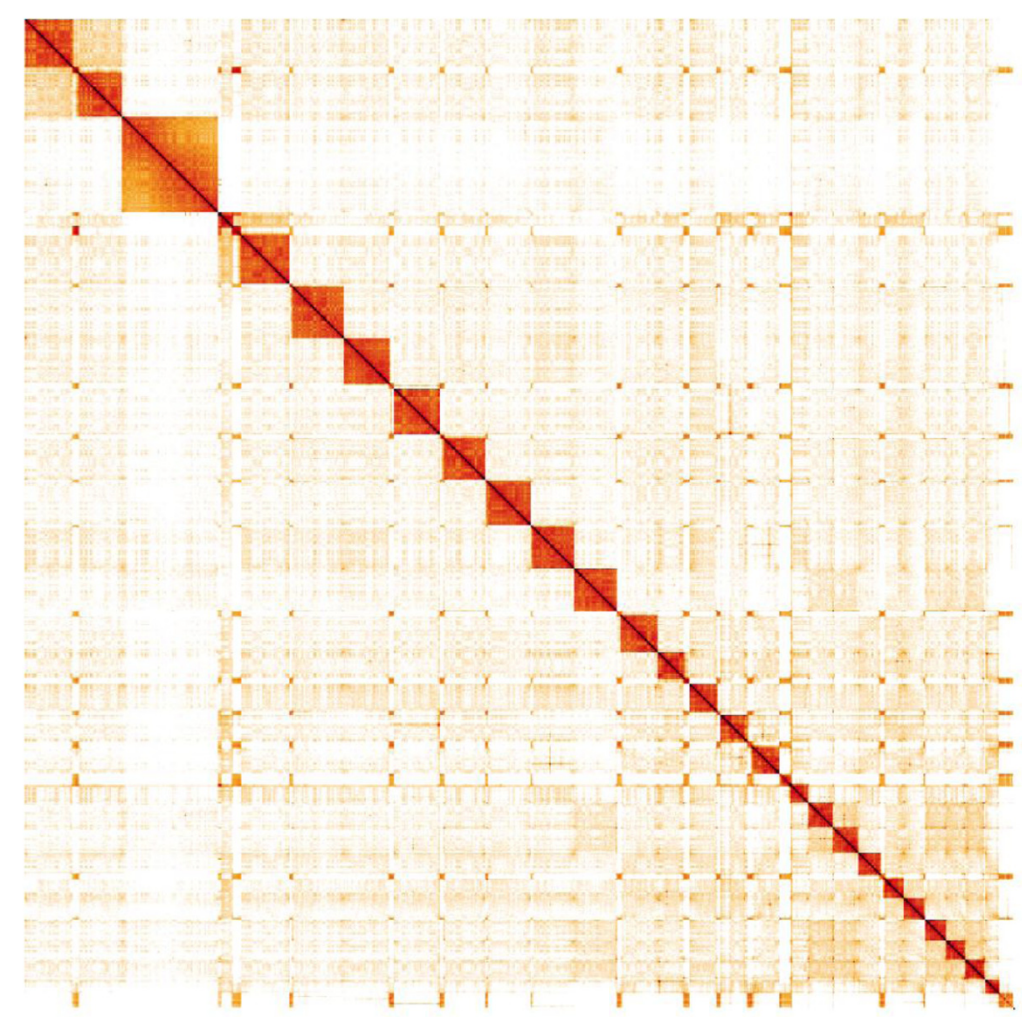

Figure 4. Genome assembly of Vespula germanica, iyVesGerm1.1: Hi-C contact map. Hi-C contact map of the iyVesCrab1.1 assembly, visualised in HiGlass. Chromosomes are shown in size order from left to right and top to bottom.

Table 2. Chromosomal pseudomolecules in the genome assembly of Vespula germanica, iyVesGerm1.1.

\begin{tabular}{|c|c|c|c|}
\hline INSDC accession & Chromosome & Size (Mb) & GC\% \\
\hline HG996528.1 & 1 & 20.39 & 35.7 \\
\hline HG996529.1 & 2 & 19.91 & 35.2 \\
\hline HG996530.1 & 3 & 14.87 & 35.8 \\
\hline HG996531.1 & 4 & 11.21 & 34.9 \\
\hline HG996532.1 & 5 & 10.58 & 35.8 \\
\hline HG996533.1 & 6 & 9.48 & 34.5 \\
\hline HG996534.1 & 7 & 9.47 & 35.8 \\
\hline HG996535.1 & 8 & 9.44 & 35.2 \\
\hline HG996536.1 & 9 & 9.01 & 35.2 \\
\hline HG996537.1 & 10 & 8.94 & 34.1 \\
\hline HG996538.1 & 11 & 8.41 & 34.8 \\
\hline HG996539.1 & 12 & 6.60 & 35.2 \\
\hline
\end{tabular}

\begin{tabular}{|c|c|c|c|}
\hline INSDC accession & Chromosome & Size (Mb) & $\mathbf{G C}$ \\
\hline HG996540.1 & 13 & 6.55 & 35.2 \\
\hline HG996541.1 & 14 & 6.12 & 34.5 \\
\hline HG996542.1 & 15 & 6.11 & 34.1 \\
\hline HG996543.1 & 16 & 5.79 & 36.3 \\
\hline HG996544.1 & 17 & 5.26 & 35.2 \\
\hline HG996545.1 & 18 & 5.24 & 34.1 \\
\hline HG996546.1 & 19 & 4.99 & 33.9 \\
\hline HG996547.1 & 20 & 4.56 & 34.8 \\
\hline HG996548.1 & 21 & 4.48 & 33.7 \\
\hline HG996549.1 & 22 & 4.34 & 33.5 \\
\hline HG996550.1 & 23 & 4.05 & 33.8 \\
\hline HG996551.1 & 24 & 3.92 & 36.0 \\
\hline HG996552.1 & 25 & 3.10 & 31.6 \\
\hline HG996553.1 & MT & 0.02 & 18.1 \\
\hline- & Unplaced & 2.97 & 43.3 \\
\hline
\end{tabular}


Table 3. Software tools used.

\begin{tabular}{|l|l|l|}
\hline Software tool & Version & Source \\
\hline Hifiasm & 0.12 & Cheng et al., 2021 \\
\hline purge_dups & 1.2 .3 & Guan et al., 2020 \\
\hline SALSA2 & 2.2 & Ghurye et al., 2019 \\
\hline Iongranger align & 2.2 .2 & $\begin{array}{l}\text { https://support.10xgenomics.com/genome-exome/ } \\
\text { software/pipelines/latest/advanced/other-pipelines }\end{array}$ \\
\hline freebayes & V1.3.1-17-gaa2ace8 & Garrison \& Marth, 2012 \\
\hline MitoHiFi & 1.0 & https://github.com/marcelauliano/MitoHiFi \\
\hline gEVAL & N/A & Chow et al., 2016 \\
\hline HiGlass & 1.11 .6 & Kerpedjiev et al., 2018 \\
\hline PretextView & $0.1 . x$ & https://github.com/wtsi-hpag/PretextView \\
\hline BlobToolKit & 2.6 .4 & Challis et al., 2020 \\
\hline
\end{tabular}

\section{Ethics/compliance issues}

The materials that have contributed to this genome note have been supplied by a Darwin Tree of Life Partner. The submission of materials by a Darwin Tree of Life Partner is subject to the Darwin Tree of Life Project Sampling Code of Practice. By agreeing with and signing up to the Sampling Code of Practice, the Darwin Tree of Life Partner agrees they will meet the legal and ethical requirements and standards set out within this document in respect of all samples acquired for, and supplied to, the Darwin Tree of Life Project. Each transfer of samples is further undertaken according to a Research Collaboration Agreement or Material Transfer Agreement entered into by the Darwin Tree of Life Partner, Genome Research Limited (operating as the Wellcome Sanger Institute), and in some circumstances other Darwin Tree of Life collaborators.

\section{Data availability}

European Nucleotide Archive: Vespula germanica (German wasp). Accession number PRJEB43741; https://identifiers.org/ena.embl/ PRJEB43741.

The genome sequence is released openly for reuse. The $V$. germanica genome sequencing initiative is part of the Darwin Tree of Life (DToL) project. All raw sequence data and the assembly have been deposited in INSDC databases. Raw data and assembly accession identifiers are reported in Table 1.

\section{Author information}

Members of the University of Oxford and Wytham Woods Genome Acquisition Lab are listed here: https://doi.org/10.5281/ zenodo. 5746938 .

Members of the Darwin Tree of Life Barcoding collective are listed here: https://doi.org/10.5281/zenodo.5744972.

Members of the Wellcome Sanger Institute Tree of Life programme are listed here: https://doi.org/10.5281/zenodo. 5744840 .

Members of Wellcome Sanger Institute Scientific Operations: DNA Pipelines collective are listed here: https://doi.org/10.5281/ zenodo.5746904.

Members of the Tree of Life Core Informatics collective are listed here: https://doi.org/10.5281/zenodo.5743293.

Members of the Darwin Tree of Life Consortium are listed here: https://doi.org/10.5281/zenodo.5638618.

\section{References}

Allio R, Schomaker-Bastos A, Romiguier J, et al.: MitoFinder: Efficient Automated Large-Scale Extraction of Mitogenomic Data in Target Enrichment Phylogenomics. Mol Ecol Resour. 2020; 20(4): 892-905. PubMed Abstract | Publisher Full Text | Free Full Text
Archer ME: Population Dynamics of the Social Wasps Vespula Vulgaris and Vespula Germanica in England. J Anim Ecol. 1985; 54(2): 473-85. Publisher Full Text

Archer ME: The World Distribution of the Euro-Asian Species of Paravespula 
(Hym., Vespinae). Entomologist's Monthly Magazine. 1998; 134: 279-83. Reference Source

Archer ME: Changes in Abundance of Vespula Germanica and V. Vulgaris in England. Ecol Entomol. 2001; 26(1): 1-7.

Publisher Full Text

Archer ME, Turner JA: The Vespoid Wasps:(Tiphiidae, Mutillidae, Sapygidae, Scoliidae and Vespidae) of the British Isles. In. Royal Entomological Society, 2014; 6(6)

Reference Source

Beggs JR, Brockerhoff EG, Corley JC, et al.: Ecological Effects and Management of Invasive Alien Vespidae. BioControl. 2011; 56(4): 505-26.

Publisher Full Text

Challis R, Richards E, Rajan J, et al.: BlobToolKit - Interactive Quality Assessment of Genome Assemblies. G3 (Bethesda). 2020; 10(4): 1361-74. PubMed Abstract | Publisher Full Text | Free Full Text

Cheng $\mathrm{H}$, Concepcion GT, Feng X, et al.: Haplotype-Resolved de Novo Assembly Using Phased Assembly Graphs with Hifiasm. Nat Methods. 2021; 18(2): 170-75.

PubMed Abstract | Publisher Full Text | Free Full Text

Chow W, Brugger K, Caccamo M, et al.: gEVAL - a Web-Based Browser for Evaluating Genome Assemblies. Bioinformatics. 2016; 32(16): 2508-10. PubMed Abstract | Publisher Full Text | Free Full Text

D'adamo P, Lozada M: Foraging Behavior Related to Habitat Characteristics in the Invasive Wasp Vespula Germanica. Insect Sci. 2007; 14(5): 383-88. Publisher Full Text

Eloff J, Veldtman R, Bulgarella M, et al.: Population Genetics of the Invasive Wasp Vespula Germanica in South Africa. Insect Soc. 2020; 67: 229-238. Publisher Full Text

Garrison E, Marth G: Haplotype-Based Variant Detection from Short-Read Sequencing. arXiv: 1207.3907. 2012.

Reference Source

Ghurye J, Rhie A, Walenz BP, et al.: Integrating Hi-C Links with Assembly Graphs for Chromosome-Scale Assembly. PLoS Comput Biol. 2019; 15(8): e1007273.

PubMed Abstract | Publisher Full Text | Free Full Text

Goodisman MA, Matthews RW, Crozier RH: Mating and Reproduction in the Wasp Vespula Germanica. Behav Ecol Sociobiol. 2002; 51(6): 497-502. Publisher Full Text

Guan D, McCarthy SA, Wood J, et al.: Identifying and Removing Haplotypic Duplication in Primary Genome Assemblies. Bioinformatics. 2020; 36(9): 2896-98. PubMed Abstract | Publisher Full Text | Free Full Text

Howe K, Chow W, Collins J, et al.: Significantly Improving the Quality of Genome Assemblies through Curation. GigaScience. 2021; 10(1): giaa153. PubMed Abstract | Publisher Full Text | Free Full Text

Hurd CR, Jeanne RL, Nordheim EV: Temporal Polyethism and Worker Specialization in the Wasp, Vespula Germanica. J Insect Sci. 2007; 7: 1-13. PubMed Abstract | Publisher Full Text | Free Full Text

Kerpedjiev P, Abdennur N, Lekschas F, et al:: HiGlass: Web-Based Visua Exploration and Analysis of Genome Interaction Maps. Genome Biol. 2018; 19(1): 125.

PubMed Abstract | Publisher Full Text | Free Full Text

Manni M, Berkeley MR, Seppey M, et al.: BUSCo Update: Novel and Streamlined Workflows along with Broader and Deeper Phylogenetic Coverage for Scoring of Eukaryotic, Prokaryotic, and Viral Genomes. $\mathrm{Mol}$ Biol Evol. 2021; 38(10): 4647-54.

PubMed Abstract | Publisher Full Text | Free Full Text

Masciocchi M, Corley J: Distribution, Dispersal and Spread of the Invasive Social Wasp (Vespula Germanica) in Argentina. Austral Ecol. 2013; 38(2): 162-68. Publisher Full Tex

Rao SS, Huntley MH, Durand NC, et al.: A 3D Map of the Human Genome at Kilobase Resolution Reveals Principles of Chromatin Looping. Cell. 2014; 159(7): $1665-80$

PubMed Abstract | Publisher Full Text | Free Full Text

Uliano-Silva M, Nunes JGF, Krasheninnikova K, et al:: marcelauliano/MitoHiFi: mitohifi_v2.0. 2021.

Publisher Full Tex 


\section{Open Peer Review}

\section{Current Peer Review Status:}

\section{Version 1}

Reviewer Report 13 September 2022

https://doi.org/10.21956/wellcomeopenres.19590.r51963

(c) 2022 Menezes R. This is an open access peer review report distributed under the terms of the Creative Commons Attribution License, which permits unrestricted use, distribution, and reproduction in any medium, provided the original work is properly cited.

\section{Rodolpho S. T. Menezes}

Universidade Federal de Santa Maria, Santa Maria, RS, Brazil

This short genome report describes the genome sequencing of the German wasp, Vespula germanica. The work begins with a background about the natural history as well as some morphological features of this social wasp are commented. The DNA extraction, sequencing, and assembly process are well described. The genome assembly reported will be interesting for further studies.

I think in the sections "Genome sequence report" and "Genome annotation report", information about the mitochondrial genome should be better described.

Keywords: Please be in alphabetic order. Change "chromosomal" to "chromosome".

Is the rationale for creating the dataset(s) clearly described?

Yes

Are the protocols appropriate and is the work technically sound?

Yes

Are sufficient details of methods and materials provided to allow replication by others? Yes

Are the datasets clearly presented in a useable and accessible format? Partly

Competing Interests: No competing interests were disclosed.

Reviewer Expertise: Phylogenomics, Phylogeography, and Cytogenetics.

I confirm that I have read this submission and believe that I have an appropriate level of 
expertise to confirm that it is of an acceptable scientific standard.

Reviewer Report 16 March 2022

https://doi.org/10.21956/wellcomeopenres.19590.r48729

(C) 2022 Brendel V. This is an open access peer review report distributed under the terms of the Creative Commons Attribution License, which permits unrestricted use, distribution, and reproduction in any medium, provided the original work is properly cited.

\section{Volker P. Brendel}

Department of Biology, Indiana University, Bloomington, IN, USA

The Data Note provides a succinct review of the genome assembly and annotation efforts by the author group concerning the German wasp. The approaches taken are standard practice and well enough documented to serve as a template for similar genome projects. All the data (raw sequence reads and derived pseudomolecules and hypothetical annotation products) are easily accessible in public databases. The genome assembly and annotation provides a starting point for further phylogenetic and functional studies not pursued in this paper - and rightly so, as such studies should encompass multiple similar data sets.

Is the rationale for creating the dataset(s) clearly described?

Yes

Are the protocols appropriate and is the work technically sound?

Yes

Are sufficient details of methods and materials provided to allow replication by others? Yes

Are the datasets clearly presented in a useable and accessible format?

Yes

Competing Interests: No competing interests were disclosed.

Reviewer Expertise: computational genomics

I confirm that I have read this submission and believe that I have an appropriate level of expertise to confirm that it is of an acceptable scientific standard. 